\title{
High resolution observations of the neutral hydrogen absorption and jets in 3C 293
}

\author{
R. J. Beswick ${ }^{1}$, A. B. Peck ${ }^{2}$, A. Pedlar ${ }^{1}$, G. B. Taylor ${ }^{3}$, \\ G. Giovannini ${ }^{4}$, J. P. Leahy ${ }^{1}$ and N. J. Jackson ${ }^{1}$ \\ ${ }^{1}$ Jodrell Bank Observatory, The University of Manchester, Macclesfield, Cheshire SK11 9DL, \\ UK email: rbeswick@jb.man.ac.uk \\ ${ }^{2}$ Harvard-Smithsonian Center for Astrophysics, SAO/SMA Project, P.O. Box 824, Hilo, \\ HI 9672, USA \\ 3. National Radio Astronomy Observatory, P.O. Box O, Socorro, NM 87801, USA \\ 4. Istituto di Radioastronomia del CNR, via Gobetti 101, 40129 Bologna, Italy
}

\begin{abstract}
We present results from new high angular resolution observations of the neutral hydrogen absorption using global VLBI, MERLIN, and VLA observations of the peculiar radio galaxy 3C 293. These results reveal extensive Hi absorption against the inner kiloparsec of the radio jet in this source over a wide range of angular scales. In additional to these sub-arcsecond resolution radio observations we also present infrared imaging of the jet.
\end{abstract}

\section{Introduction}

At the present time, most high angular resolution studies of powerful active galaxies have concentrated upon investigating the consequences of the activity (e.g. the synchrotron emission such as radio jets), rather than the cause of the activity (e.g. cold neutral and molecular gas that fuels the nuclear activity). This has been a direct consequence of observational constraints resulting from the relatively small collecting areas of the current generation of $\mathrm{mm}$-wavelength aperture synthesis instruments and the surface brightness sensitivity of decimetre wavelength interferometers, precluding observations of cold thermal gas emission at angular resolutions of $\lesssim 1^{\prime \prime}$. However, using current radio aperture synthesis techniques it is possible to observe cold gas, via transitions such as $\mathrm{HI}$, $\mathrm{OH}$ and $\mathrm{H}_{2} \mathrm{CO}$, in absorption against the bright background radio continuum of some galaxies, on sub-arsecond angular scales.

In this proceedings we will briefly summarise new high resolution (milliarcsecond) observations of the inner jet (consequences of the activity) and neutral hydrogen (causes of the activity) absorption in the radio galaxy $3 \mathrm{C} 293$.

3C 293 is a nearby $(\mathrm{D}=180 \mathrm{Mpc}$, implying 1 mas $=0.815 \mathrm{pc})$ radio galaxy, associated with the peculiar elliptical host galaxy VV5-33-12. On scales of several tens of kiloparsecs, the radio structure of 3C 293 resembles a moderately large two-sided FR-II radio galaxy with an unusually bright steep-spectrum extended core component (Akujor et al. 1996; Beswick, Pedlar \& Holloway 2002). When observed with sub-arcsecond angular resolution, this central core region resolves into a complex, asymmetric east-west radio jet, which is embedded in the host galaxy. Against this inner jet component deep and very broad Hi absorption is detected (Haschick \& Baan 1985; Morganti et al. 2003; Beswick et al. 2002, 2004 and references therein). This absorption has been used to trace the neutral gas dynamics at high angular resolution toward the centre of this galaxy. 

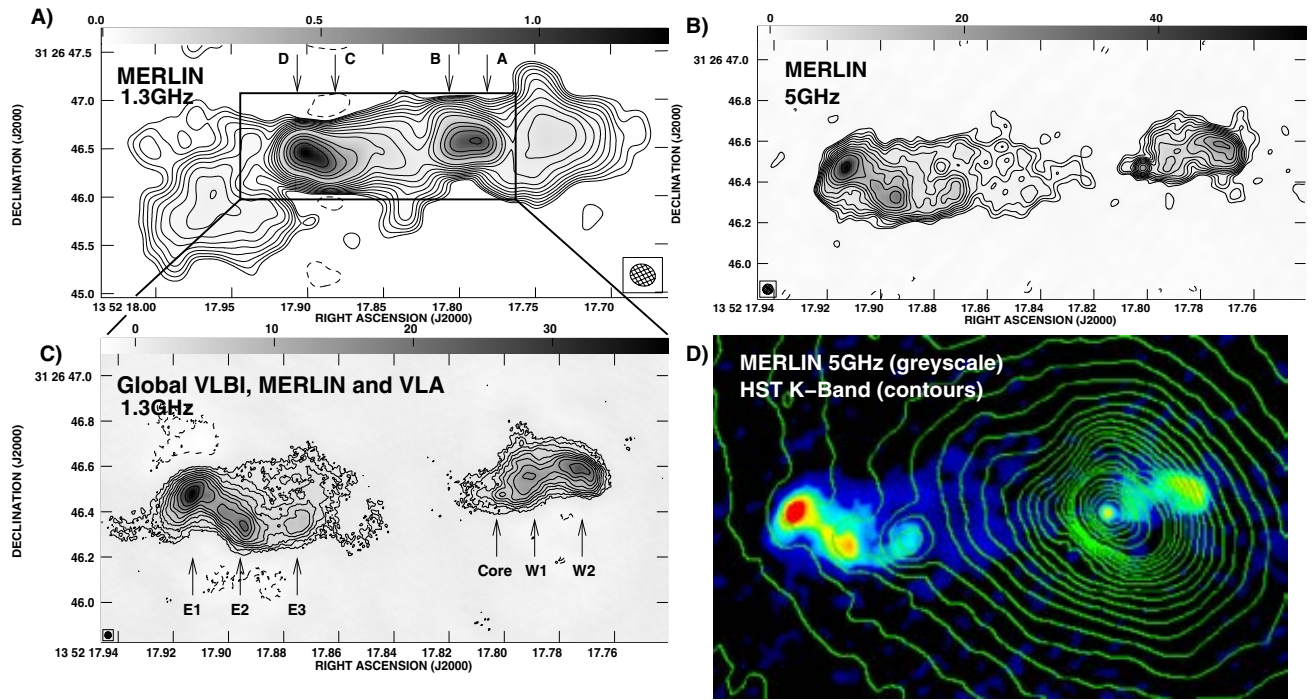

Figure 1. Montage of multi-frequency radio and IR continuum images of the inner jet of 3C 293. A) 200 mas angular resolution $1.3 \mathrm{GHz}$ MERLIN image. Contour levels are $\sqrt{2}$ times $0.5 \mathrm{mJy} \mathrm{bm}^{-1}$. B) 50 mas angular resolution $5 \mathrm{GHz}$ MERLIN image. Contour levels are $\sqrt{2}$ times $0.74 \mathrm{mJy} \mathrm{bm}^{-1}$. C) 30 mas angular resolution $1.3 \mathrm{GHz}$ combined global VLBI, MERLIN and VLA image. Contour levels are $\sqrt{2}$ times $1.3 \mathrm{mJy} \mathrm{bm}^{-1}$. D) False-colour $5 \mathrm{GHz}$ radio image with superimposed contoured K-band HST image. Note that there are several knots of infrared jet emission coincident with the eastern radio hotspots.

\section{Observations}

The Multi-Element Radio Linked Interferometric Network (MERLIN), in the UK, has been used to observe both neutral hydrogen absorption and the radio continuum of 3C 293 (Beswick et al. 2002), these data have been combined with Very Large Array (VLA) (including the Very Long Baseline Array (VLBA) antenna at Pie Town) and global Very Long Baseline Interferomtry (VLBI) observations (Beswick et al. 2004) in order to produce a high angular resolution and high fidelity image of the inner jets and the Hi absorption in this source (see Fig. 1 and 2). These data have been supplemented by further MERLIN $5 \mathrm{GHz}$ and Hubble Space Telescope (HST) observations of the host galaxy and jet in 3C 293 (Leahy et al. 1999; Beswick et al. , in prep.).

\section{Results \& discussion}

\subsection{The jet emission}

The inner few kiloparsecs of the jet of 3C 293 are shown at a variety of angular resolutions and frequencies in Fig. 1. As can be seen on these scales the radio jet in 3C 293 follows an almost east-west orientation. The orientation of these inner hotspots and jet are misaligned by $\sim 45^{\circ}$ to the large-scale FR-II radio lobes (Bridle et al. 1981; Beswick et al. 2004). In the $0^{\prime \prime} 2$ angular resolution MERLIN $1.3 \mathrm{GHz}$ observations, the inner jet of $3 \mathrm{C} 293$ shows at least four distinct radio components (labelled A, B, C and D in Fig. 1a) with two more diffuse lobe-like components extending on either side of the central region. At this resolution and frequency it is unclear which radio continuum component is coincident with the AGN. Whereas in the $5 \mathrm{GHz}$ MERLIN observations of the inner jet (Fig. 1b), the steeply inverted core component is clearly evident, as is the complexity of the inner radio jet. Fig. 1c shows the radio continuum structure of the inner part of 

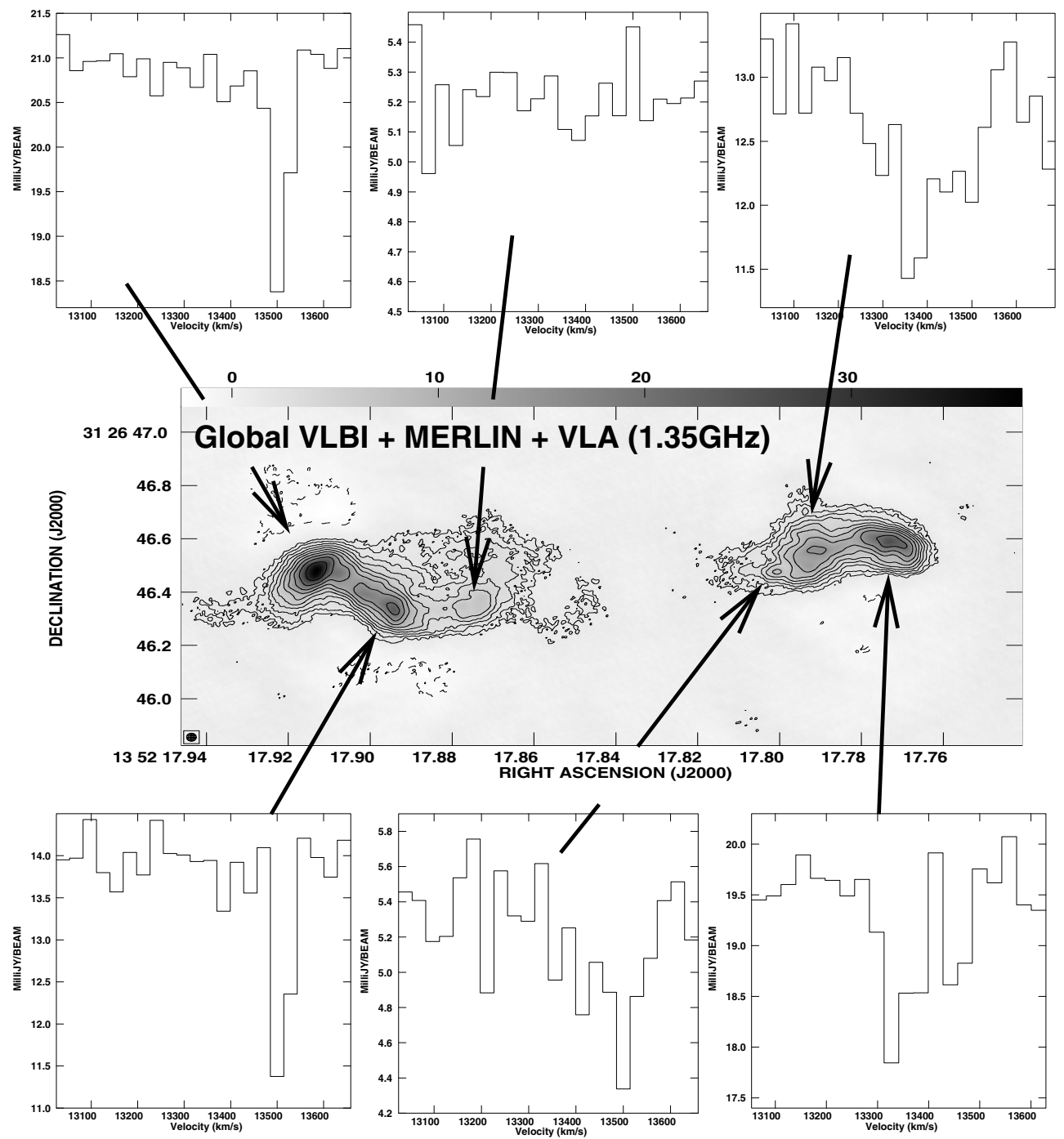

Figure 2. Combined VLBI, MERLIN and VLA image of the $1.3 \mathrm{GHz}$ radio continuum convolved with a circular 30 mas synthesised beam with selected HI absorption spectra.

the MERLIN image at $1.3 \mathrm{GHz}$. In this high resolution image, the inner kiloparsec of the radio jet in 3C 293 can be seen to deviate significantly from its east-west trajectory. The continuum components $\mathrm{A}, \mathrm{B}, \mathrm{C}$ and $\mathrm{D}$, which are barely resolved in the MERLIN image, are composed of numerous compact, bright knots of radio emission tracing the path of the radio jet. It is likely that the deviations observed in the jet inner structure of 3C 293 are symptomatic of the jet and the ISM interacting with each other (see Morganti, this proceedings; Beswick et al. 2004).

\subsection{Neutral hydrogen absorption}

Using the sub-arsecond angular resolution of MERLIN at a wavelength of $21 \mathrm{~cm}$, strong HI absorption has been detected against the entire radio jet (see Beswick et al. 2002). When these data are combined with global VLBI and VLA data, not only the jet, but also the HI absorption can be mapped on milliarcsecond angular scales (see Fig. 2). The Hi absorption 
distribution can be broadly split into two components; a broad components primarily observed against the western counter-jet and core region, and a narrow component seen against the eastern jet. In this MERLIN study the Hi opacity detected against the jet is found to be highly correlated with the dust lanes, with the narrow Hi absorption component tracing the dust distribution observed in WFPC2 F702W snapshot images. This is confirmed in the higher resolution VLBI studies (Beswick et al. 2004). In these higher resolution studies this component is also shown to follow a small velocity gradient of $\sim 50 \mathrm{~km} \mathrm{~s}^{-1} \operatorname{arcsec}^{-1}$, which is consistent with the galactic rotation of the host galaxy.

The broadest absorption is detected against the core region and the counter-jet (core, $\mathrm{W} 1$ and W2 in Fig. 1c). This absorption is more complex and is primarily composed of a double absorption line (see Fig. 2). Although, especially in the VLBI data, much of the illuminating background radio continuum is resolved away, this complex absorption does show some velocity structure on linear scales ranging from $\sim 1 / 2 \mathrm{kpc}$ to $\sim 50 \mathrm{pc}$. These absorbing structures can be interpreted as either, consistent with two unrelated HI gas systems along the line of sight to the core, or with a steep velocity centred upon the core. If the latter is assumed to be correct then the mass enclosed by this rotating gas ring would be at least $1.7 \times 10^{9} \mathrm{M}_{\odot}$ within $400 \mathrm{pc}$ of the core.

\section{Acknowledgements}

RJB acknowledges PPARC support. The VLA and VLBA are operated by the National Radio Astronomy Observatory (NRAO). NRAO is a facility of the National Science Foundation operated under cooperative agreement by Associated Universities, Inc. MERLIN is a national facility operated by the University of Manchester on behalf of PPARC in the UK.

\section{References}

Akujor, C. E., Leahy, J. P., Garrington, S. T., Sanghera, H., Spencer, R. E., \& Schilizzi, R. T. 1996, MNRAS, 278, 1

Beswick, R. J., Pedlar, A., \& Holloway, A. J. 2002, MNRAS, 329, 620

Beswick, R. J., Peck, A. B., Taylor, G. B., \& Giovannini, G. 2004, MNRAS, in press (astro$\mathrm{ph} / 0404295)$

Bridle, A. H., Fomalont, E. B., \& Cornwell, T. J 1981, AJ, 86, 1294

Haschick, A. D., \& Baan, W. A. 1985, ApJ, 289, 574

Leahy, J. P., Sparks, W. B., \& Jackson, N. J. F. 1999, AAS, 194, 73.04

Morganti, R., Oosterloo, T. A., Emonts, B. H. C., van der Hulst, J. M., \& Tadhunter, C. N. 2003, ApJ, 593, L69 